\title{
Erratum to: Irreversibility Line Measurement and Vortex Dynamics in High Magnetic Fields in Ni- and Co-Doped Iron Pnictide Bulk Superconductors
}

\author{
Martin Nikolo $^{1}$ - John Singleton ${ }^{2}$ Vivien S. Zapf ${ }^{2}$ - Anders Hansen ${ }^{2} \cdot$ Jianyi Jiang $^{3}$. \\ Jeremy Weiss ${ }^{3} \cdot$ Eric Hellstrom $^{3}$
}

Published online: 30 September 2016

(C) Springer Science+Business Media New York 2016

Erratum to: J Supercond Nov Magn (2016)

DOI 10.1007/s10948-016-3628-6

The original version of this article unfortunately omitted one of the authors' names. We add Anders Hansen as one of the authors.

The online version of the original article can be found at http://dx.doi.org/10.1007/s10948-016-3628-6

\footnotetext{
Martin Nikolo

nikolom@slu.edu

1 Department of Physics, Saint Louis University, St. Louis, MO 63103, USA

2 National High Magnetic Field Laboratory, Los Alamos National Laboratory, Los Alamos, NM 87545, USA

3 Applied Superconductivity Center, National High Magnetic Field Laboratory, Florida State University, Tallahassee, FL 32310, USA
} 